\title{
An Ultra-Low Power Wake-Up Receiver for Real-time constrained Wireless Sensor Networks
}

\author{
Sadok Bdiri†, Faouzi Derbel ${ }^{\top}$ \\ Leipzig University of Applied Sciences, Wachter Str. 13, 04107, Leipzig, Germany \\ †sadok.bdiri@htwk-leipzig.de \\ Ifaouzi.derbel@htwk-leipzig.de
}

\begin{abstract}
Most Wireless Sensor Networks (WSNs) are considered out of reach when deployed in harsh environment. Energy consumption is a critical feature as these networks incorporate battery-powered devices known as wireless sensor nodes (WSn). Typically, the majority of applications don't need from nodes to remain active all the time. However, what is called idle listening waste most of the energy since the radio transceiver is constantly active. In case of real-time application, the energy-hungry chip remains in idle state, consuming power when communication traffic is considerably low. On the other hand, putting it into sleep state disconnects the node from the network. To cope with such challenge, an ultra-low power radio receiver referred as Wake-Up Receiver (WuRx) handles the idle listening while keeping the main radio completely off. The WuRx consumes much less power than the main transceiver. Embedding such device enables more event-triggered applications where real-time behavior is critical and longer lifetime is mandatory. The WuRx suffers from mediocre sensitivity and signal selectivity as it includes the minimum number of active components in order to remain within the power budget. In this paper, we introduce an ultra-low power WuRx that drains less than $3 \mu \mathrm{A}$ with a sensitivity $-60 \mathrm{dBm}$. The device can decode a 16-bit wake-up packet (WuPt) with minimally $1 \%$ of PER in nearly $10 \mathrm{~ms}$.
\end{abstract}

\section{Index Terms}

Wireless sensor networks, radio, Wake-Up receiver, power consumption, sensitivity, event-triggered, on-demand.

\section{INTRODUCTION}

The development of low-power radio has enabled new applications such as wireless sensor networks (WSNs) and wireless body area networks (WBANs). These applications has been covering scientific, industrial and medical fields. These networks include devices which are invited to monitor the occurrence of ambient data and transmit them in untethered manner. Over and above that, one of the criteria that defines such networks is the low-power consumption budget of batter-powered sensor nodes, thus longer operational lifetime. One of the reasons behind is the deployment cost of these networks because, in general, a network will include tenth to thousands of nodes. Besides, in specific applications like hazardous work environments, nodes are unreachable in case of battery or entire node replacement. Since radio transceivers energy consumption has a direct impact on the overall system lifetime, works have been focused on managing the activity of these radios or attempts on reducing power consumption in further designs.
Despite the extremely low-power constraint, the lifetime condition required for certain applications must be fulfilled. As a software-based solution, communication protocols that use dutycycled radio receivers scheme decrease energy consumption. Although, a fact of higher latency is paid when the radio is in sleep state for long period of time. However, real-time applications scheme must operate with very low communication latency. At this point, low-power radios known as wake-up receivers (WuRx) are subject to replace main transceivers for continuous idle listening task while consuming much less power. The main feature of WuRx is to remain alwayson in order to receive an decode a special signal with unique address. Afterwards, an interrupt signal will be forwarded to the sleeping node to wake-up and initiate the communication between receiver and sender nodes. The reduced power consumption in WuRxs is paid in terms of other performance factors like sensitivity and circuitry complexity. Previous works tend to achieve an 
optimum point within such tradeoff.

In this work, a low-power sensor node equipped with an ultra-low-power WuRx with at least $-56 \mathrm{dBm}$ sensitivity is introduced. The WuRx performs in sub-GHz ISM band with a latency of $10.36 \mathrm{~ms}$. After some previous work in Section II, in section III, the system design is presented. Hardware implementation and System evaluation are described in Section IV. Finally, conclusions and final comments will be given in Section V.

\section{Previous Work}

Realizing that the possibility of lowering the power consumption to an extreme low level is an idea which has been conducted by many contributors along the years. It starts by wondering on how to detect an RF signal and how sensitive and power-hungry an RF detector can be. We can find sophisticated techniques which achieve excellent detection sensitivity, but they dissipate more energy. As a commonly pursued strategy, the focus on designing Wake-Up radios using passive detection to guarantee the low power budget is followed by attempts on enhancing sensitivity and other features. A passive radio receiver architecture was firstly introduced by $\mathrm{Gu}$ and Stankovic [2]. The device uses the RF signal to raise a voltage level which will behave as an interrupt to the Microcontroller (MCU). As an addressing technique, each WuRx receives a WuPt on different frequency [1][2]. Undoubtedly, such hardware design increases complexity for both Wake-Up Transmitter (WuTx) and WuRx.

Recent Works [3][6] have focused on improving a single feature at the price of another, a tradeoff of power consumption and sensitivity. In real world, most applications require a practical balance of this trade-off in order respond to the desired performance. Publication like [4] and [5] considered coping with that challenge. The efforts can be seen in the introduced WuRxs with $-52 \mathrm{dBm}$ and $-72 \mathrm{dBm}$ sensitivity level and consuming $8.1 \mu W$ and $2.4 \mu W$ respectively.

The WuRx from Gamm et Al. [4] mainly downconverts the RF signal from $868 \mathrm{MHz}$ to 125 $\mathrm{KHz}$ using zero bias Schottky diodes, which are configured as an AC-DC rectifier. The incoming signal is OOK modulated where from transmitter side, a digital bit ' 1 ' is encoded by transmitting the RF carrier, while a ' 0 ' is basically the absence of the carrier. The period between the encoded digits presents the frequency of the rectified signal. It is, then, fed to a low frequency WuRx AS3932. The device can correlate up to 32 -bit OOK modulated address with a sensitivity of $100 \mu V_{R M S}$ as well as a high input impedance of $2 M \Omega[8]$. In the end, an interrupt is generated to $\mathrm{MCU}$ once the address matches the one saved the in AS3932's registers.

\section{SyStem DEsign}

In this section, the design analysis of all blocks of WuRx are discussed. The different parts of WuRx circuit are illustrated in Fig 1.

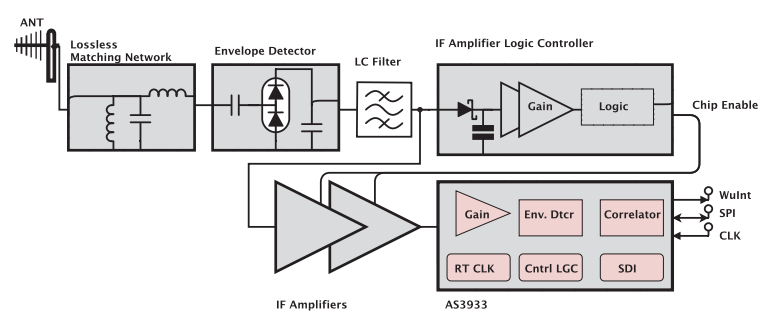

Fig. 1: Wake-Up Receiver block diagram

\section{A. Envelope Detector}

The overall performance of WuRx severely depends on the envelope detector characteristics. For such reason, components are carefully chosen in order to achieve the desired objectives of the introduced design. The envelope detector detects the incoming signal by means of a passive receiver, notably using Schottky diodes HSMS-2852 [7]. The latter are zero biased and they provide fast switching. The diodes are optimized to be used with a input power less than $-20 \mathrm{dBm}$ and below a frequency of $1.5 \mathrm{GHz}$. The detector is configured as a Greinacher voltage doubler. Such configuration provides higher output voltage, which is considered as an advantage over a signal diode configuration. The voltage doubler acts also as a halfwave rectifier in order to perform an RF-to-DC conversion. Conversely, the envelope detector is able to interpret an incoming OOK modulated signal. As mentioned earlier, the output signal swings between two voltage levels according to input signal. Accordingly, as described in Fig. 2 the resulted output is a square signal with a user-selected low frequency equal to ON/OFF frequency of OOK signal.

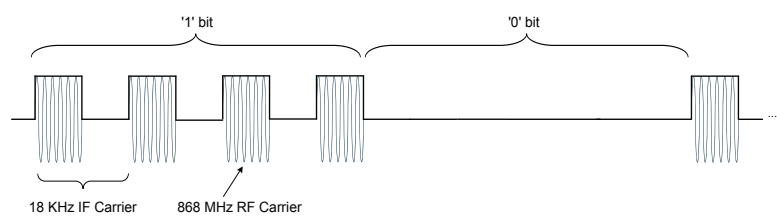

Fig. 2: AS3933 Wake-Up packet structure 
The LC filter is used to smooth a signal to obtain a better DC signal. The impact of the filter is more important at low input power signals.

\section{B. Intermediate Frequency Amplifiers}

The AS3933 has a voltage sensitivity of $100 \mu V_{R M S}$ [9]. The HSMS-2852 schottky diodes are able to sense a signal down to $-57 \mathrm{dBm}$ above the noise floor (NF) [7]. An amplification needs to be performed in order to shift a smallsignal voltage level above minimum detectable voltage level of the AS3933.

Small-signal high gain amplification within the micro-power budget at a frequency of few $\mathrm{KHz}$ faces several challenges using ultra low-power amplifiers. However, higher performance devices are subject to accomplish the desired performance. On the other hand, the nature of on-demand/real-time applications of WSNs and WuRx depicts that generally, nodes are inactive. Such criteria is used as a benefit for design if amplifiers can function only during the WuPt decoding and shutdown during channel monitoring of WuRx. The switch between both of these modes requires from the amplifiers to include a shutdown feature. Although the incorporated amplifiers have high energy usage, lowering their duty-cycle offers a very low overall energy consumption which meets the power efficiency requirements. The overall current consumption of IF amplifiers $I_{A m p}$ is calculated in the following expression.

$$
I_{A m p}=D I_{A m p \_ \text {Active }}+(1-D) I_{\text {Amp_Sleep }}
$$

Where, $I_{A m p \_ \text {Active }}$ is the power consumption of the amplifier when active for a certain time $t_{\text {Active }}, I_{\text {Amp_Sleep }}$ when sleep/shutdown for $t_{\text {Sleep }}$ and the applied duty-cycle $D=\frac{t_{\text {Active }}}{t_{\text {Active }}+t_{\text {Sleep }}}$.

The proposed design requires high precision and high efficiency amplifiers with optimal frequency/performance ratio. Therefore, instrumentation amplifiers are chosen to be incorporated in the current WuRx design since they are known for their low offset voltage and precision in high gain applications. The proposed WuRx adopts 3 stages of the instrumentation amplifier MAX4461T where the closed-loop gain is $20 \mathrm{~dB}, I_{\text {Amp_Active }}=700 \mathrm{nA}$, $V_{O S}=750 \mu \mathrm{V}$ and $I_{\text {Amp_Sleep }}=10 \mathrm{nA}$ [10].

The MAX4461 has to last in active mode for a minimum amount of time to fully amplify the WuPt signal. The condition is expressed as follows:

$$
t_{W u P t} \leq t_{\text {Active }}
$$

Where $t_{W u P t}=10.36 \mathrm{~ms}$ and $t_{\text {Active }}=15 \mathrm{~ms}$ is the chosen amount of the IF amplifiers activity time. Furthermore, The average current consumption increases by increasing the number of WuPt decoding times over a certain period of time. The relation is plotted in 3 .

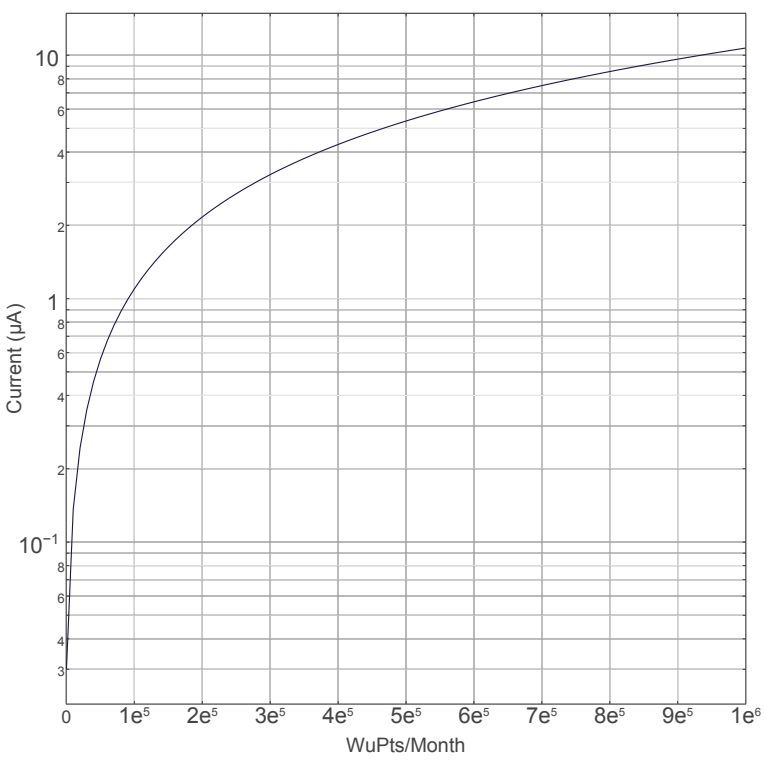

Fig. 3: Overall IF amplifier power consumption deviation according to number of received

WuPt per month

Once a WuPt is received, the IF amplifiers switch to active mode by means of an incoming signal at the SHDN pin. The signal is a LOW-toHIGH pulse which lasts $t_{\text {Active }}$. A current drain of $I_{A m p}=137 n A$ for a number of received packet of $10 e^{4}$ per month satisfies the required energy budget of the WuRx. However, $10 e^{4}$ of packet per month is considered very high traffic based on the nature of on-demand or real-time applications.

\section{Intermediate Frequency Logic Controller}

The IF amplifier activates only when SHDN is driven HIGH by means of circuitry named Intermediate Frequency Logic Controller (IFLC). The idea is to generate a low-to-high pulse using the received WuPt signal. The circuitry relies only on the carrier burst data since it's set unchanged in every WuPt. Accordingly, the preamble and data pulses are not taken into consideration to generate the wanted SHDN signal. The carrier burst is an $18 \mathrm{KHz}$ square-wave signal which lasts $t_{C} \simeq \frac{1}{2} t_{W u P t}$ as can be seen in the simulation (Fig. 4 (a)). The frequency is chosen according to the AS3933 requirements. In the current design, IFLC is configured to perform a frequency down-conversion from 18 
$\mathrm{KHz}$ to $33 \mathrm{~Hz}$. The rectification process in the IFLC is done by means of peak detector then amplified by two stages of operational amplifiers (opAmp) ISL28194 [11]. Therefore, the obtained signal is a rectified voltage of the carrier burst. A simulation is illustrated in Fig. 4 (b).
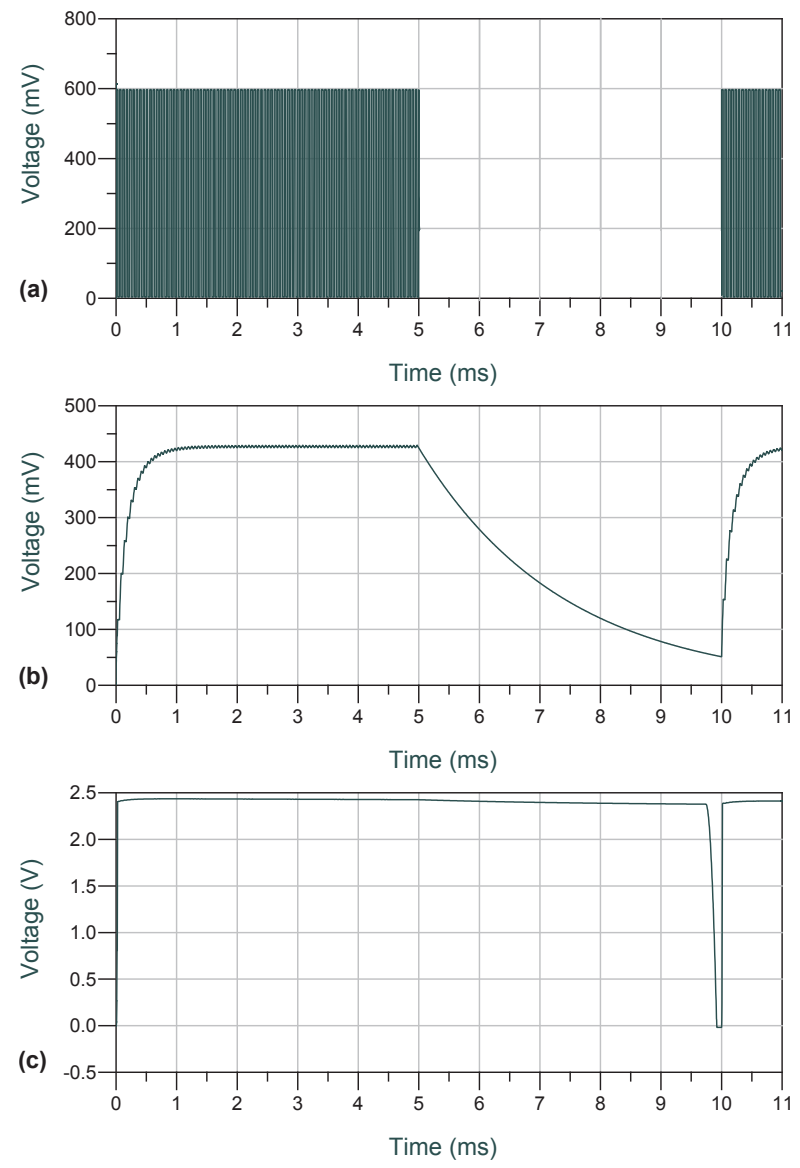

Fig. 4: (a) $18 \mathrm{KHz}$ WuPt (carrier burst) simulation, (b) peak detector waveform. (c) Final generated pulse by IFLC.

The amplified pulse's amplitude varies proportionally with the input power of the WuRx. However, the logic level of SHDN implies that A $\mathrm{HIGH}$ signal must have an amplitude of minimum $0.7 V_{c c}$ and LOW signal has to be maximum $0.3 V_{c c}$. A comparator is incorporated to guarantee such logic control. The SHDN pulse is illustrated in Fig. 4 (c).

The opAmp consumes $I_{O p A m p}=330 n \mathrm{~A}$ and the comparator TLV3691 consumes $I_{C m p}=$ $75 n A$ with a maximum propagation delay $t_{P D}=$ $45 \mu s$ [12]. The overall power consumption of the IFLC is calculated in the following expression.

$$
I_{I F C L}=2 I_{O p A m p}+I_{C m p}=735 n A
$$

\section{System Evaluation}

In this section, an experimental evaluation for the presented WuRx design is carried out.
Different test beds are set to fill in a complete survey of measurements. A developed proof of concept can be seen in Fig. 5 .

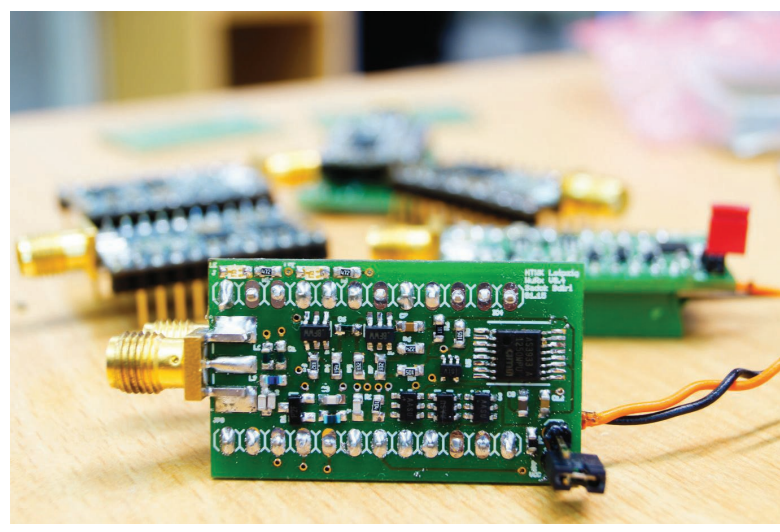

Fig. 5: Assembled WuRx PCB and attached to a host node

\section{A. Hardware Implementation}

The manufactured PCB is a 2-layer $1.55 \mathrm{~mm}$ FR4 substrate with $35 \mu \mathrm{m}$ copper width. The WuRx is interfaced with the host nodes through 24 pins. Theoretical load impedance of the WuRx using the available diodes model in ADS from Agilent [13] is $Z_{\text {load }}=33.6-j 392 \Omega$. An impedance matching network is essential to match the circuit to $50 \Omega$ system for maximum power transfer between source and load.

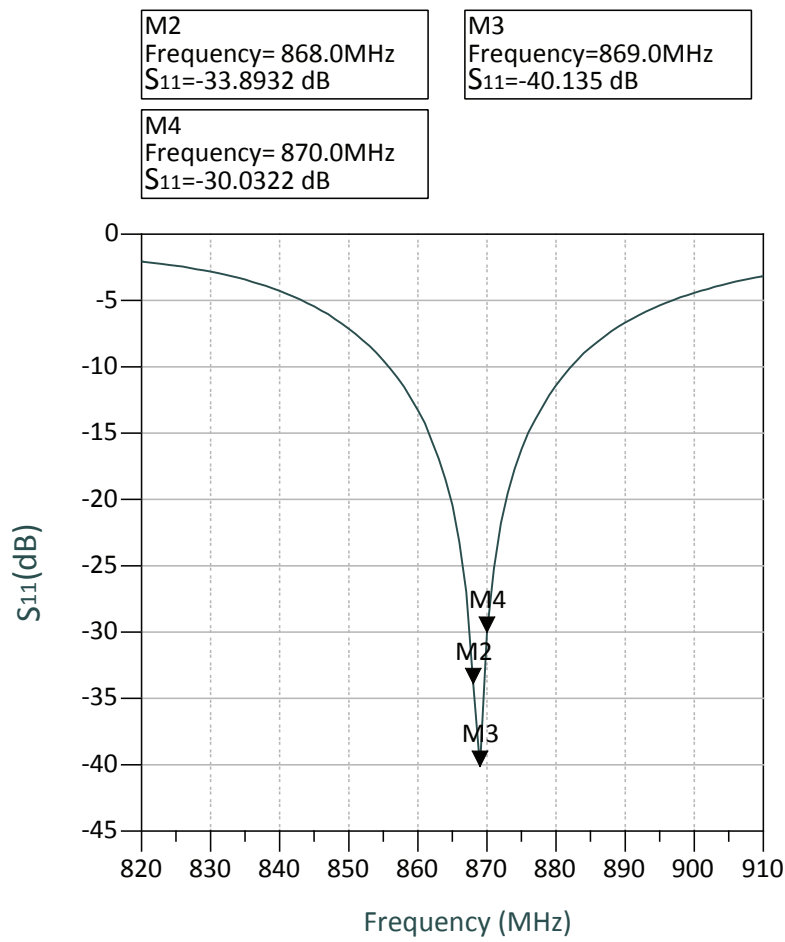

Fig. 6: Measurement of reflection coefficient $\left(S_{11} / \Gamma\right)$ at the input of the WuRx 
According to the manufacturer, the impedance values of diodes change with every released product thus deriving from a theoretical load impedance. For such reason, $Z_{\text {load }}$ is measured by means of a network analyzer to be able to determine the appropriate impedance matching network. However, components trimming was necessary to perfectly match the WuRx. A measurement of the reflection coefficient $S_{11}$ at the WuRx input on log-magnitude plot provided in Fig. 6.

Changing the operation frequency of the WuRx within $[867,870 \mathrm{MHz}]$ doesn't alter on the performance of the circuit. Although, it is found as benefit as frequency can be changed freely within a certain bandwidth. An example could be the use of one channel for WuRx and another channel for normal communication between nodes to avoid collision or interference.

\section{B. Sensitivity and Range}

One of the main objectives of designing the WuRx is to achieve at least $-55 \mathrm{dBm}$ of sensitivity as well as a tolerated performance of $1 \%$ of PER. In order to retrieve experimental measurement of the WuRx's sensitivity, a WuTx is connected to the WuRx with an attenuator in between. The WuRx is configured to transmit 100 WuPts with a transmission power $P_{T x}=$ $3 \mathrm{dBm}$. Upon reception of a WuPt, the decoder interrupts the MCU resulting in blinking blue LED. A step further using the attenuator, the transmission power is attenuated until no interrupt is induced to the MCU. A successful interrupt can be seen till an attenuation level of $63 \mathrm{~dB}$. Now, a spectrum analyzer is connected to the attenuator instead of the WuRx. At $868.01 \mathrm{MHz}$, the measured signal amplitude varies within $[-60.5,-60] \mathrm{dBm}$ with the same attenuation level. Therefore, the WuRx's minimum sensitivity is considered $P_{S}=-60 \mathrm{dBm}$. A lineof-sight range measurements between WuTx and WuRx with different transmission power is provided in Table I.

TABLE I: Range test at different WuTx transmission power

\begin{tabular}{|c||c|}
\hline Transmission Power $[\mathrm{dBm}]$ & Distance $[\mathrm{m}]$ \\
\hline \hline-10 & 8.3 \\
\hline 0 & 26.2 \\
\hline 3 & 37 \\
\hline 10 & 82.5 \\
\hline 11 & 92.9 \\
\hline
\end{tabular}

\section{Power Consumption}

Energy usage severely characterize a WuRx design. With that in mind, an energy usage profile analysis is performed on a host node with an embedded WuRx. The measurement covers possible system operational modes. Furthermore, Table II summarize the current consumption of each block in the WuRx for different operation modes.

TABLE II: WuRx current consumption in different operation modes

\begin{tabular}{|l|c|c|}
\hline Components & Monitoring $[\mu A]$ & Decoding $[\mu A]$ \\
\hline \hline Envelope Detector & 0 & 0 \\
\hline IFLC & 0.69 & 0.735 \\
\hline IF Amplifiers & 0.03 & 2090 \\
\hline AS3933 & 1.81 & 8 \\
\hline \hline WuRx & 2.53 & 2099 \\
\hline
\end{tabular}

The WuPt power consumption is crucial when decoding a WuPt. Since WuPts are sent only at event-triggering trend, the total duration in which the WuRx is decoding a WuPt is significantly less than the duration of channel monitoring. For evaluation purposes, the WuRx's activity time is set $t_{\text {Active }}=35 \mathrm{~ms}$. From (1) the average current consumption of the WuRx $I_{W u R x}$ is calculated in the following expression.

$$
I_{W u R x}=I_{C M}+N_{P t} \frac{t_{\text {active }}}{t_{\text {total }}}\left(I_{D}-I_{C M}\right)
$$

Where $I_{C M}$ and $I_{D}$ are the current consumptions of WuRx in channel monitoring and WuPt decoding states respectively. In this case, $N_{P t}$ is number of received WuPt per $t_{\text {total }}$. The resulting relation is plotted in Fig. 7.

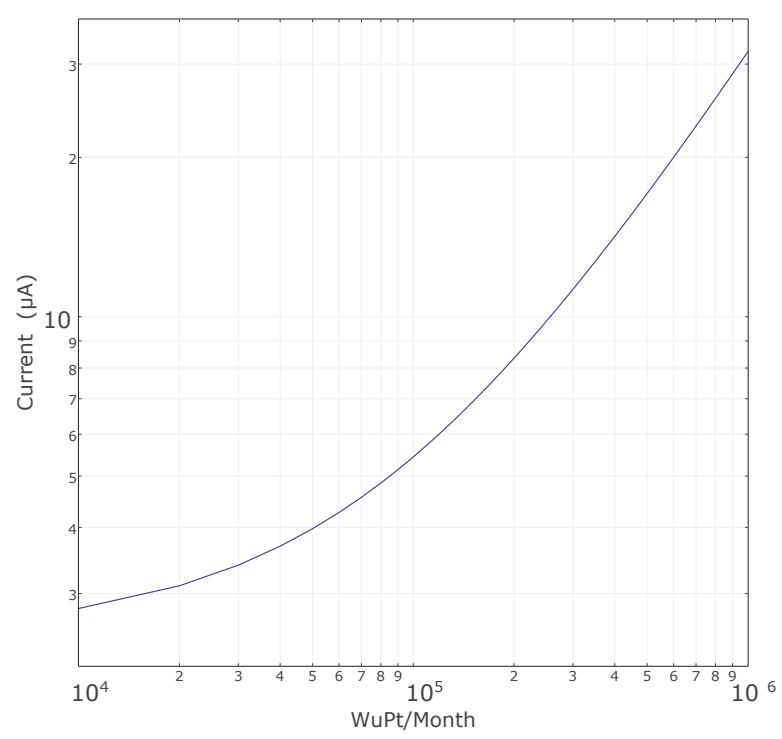

Fig. 7: Average WuRx current usage as a function of number of received WuPt per month

For a $10^{4}$ received WuPts packets in one month the average current consumption is $I_{W u R x}=2.76 \mu \mathrm{A}$ 


\section{CONCLUSION}

An ultra-low power for Real-time constrained wireless WSNs is introduced. The paper describes the rationale behind the system design and the protocol details of wake-up receiver. A novel WuRx architecture is adopted by taking the benefit from communication scheme of eventtriggering application. Such architecture makes it possible to enhance the WuRx sensitivity while remaining in the micro-power budget. The current design is able to achieve a sensitivity of $-60 \mathrm{dBm}$ while consuming $2.53 \mu \mathrm{A}$ of current in channel monitoring mode and able to decode a 16-bit wake-up pattern. At the time the paper is written, previous designs based on off-theshelf components didn't achieve a better sensitivity/energy usage ratio. At $10 \mathrm{dBm}$ of WuPt transmission power, a communication range of nearly $82 \mathrm{~m}$ in line-of-sight scheme is observed. Such coverage enables most of the on-demand WSNs applications. Further revisions will incorporate an MCU and transceiver on the same board.

\section{ACKNOWLEDGEMENTS}

This work has been supported by the Autarkic Intelligent Sensor Network For Production (AIS) project within the sub-project Energyefficient and reliable wireless sensor communication (EMT). The project is funded by the European Science Foundation (ESF).

\section{References}

[1] W. Che D. et al., "CMOS passive wake-up circuit for sensor network applications", in CiCC, 2010 IEEE. San Jose, CA. pp 1, Sept 2010.

[2] L. Gu and J.A. Stankovic, Radio-triggered wake-up capabilities for wireless sensor networks, in RTAS 2004. pp27. July 2004.

[3] N. M. Pletcher, S. Gambini, and J. Rabaey. A $52 \mu \mathrm{W}$ Wake-Up Receiver With $-72 \mathrm{dBm}$ Sensitivity Using an Uncertain-IF Architecture. In IEEE Journ. Of solid-state circuits, 44(1):269-280, Jan. 2009.

[4] G. U. Gamm, M. Sippel, M. Kostic, and L. M. Reindl. Low Power Wake-up Receiver for Wireless Sensor Nodes. In ISSNIP, pp. 121-126, 2010.

[5] C. Hambeck, S. Mahlknecht, and T. Herndl. A 2.4 $\mu W$ Wake-up Receiver for Wireless Sensor Nodes with $-71 \mathrm{dBm}$ Sensitivity. 2011.

[6] S. Marinkovic and E. Popovici. Nano-Power Wireless Wake-Up Receiver With Serial Peripheral Interface. In IEEE Jour. on selected areas in comm., 29(8):1641 1647, Sept. 2011.

[7] HSMS285x-Series Datasheet., Avago Technologies US.http://www.avagotech.com/docs/AV02-1377EN

[8] $15-150 \mathrm{KHz}$ AS3932 3D Low Frequency Wakeup Receiver, revision $1.2 \mathrm{http}: / /$ ams.com/chi/content/download/ 23636/413647/file/AS3932 Datasheet EN v2.pdf

[9] $5-150 \mathrm{KHz}$ AS3933 3D Low Frequency Wakeup Receiver, revision 1.2. http://www.mouser.com/ds/2/588/ AS3933 Datasheet EN v2-480979.pdf

[10] MAX4461 Precision Instrumentation Amplifier, revision 1.0. http://datasheets.maximintegrated.com/en/ds/ MAX4661-MAX4663.pdf
[11] Ultra-Small, $330 n A$ and $1 \mu A$ Single Supply, Rail-to-Rail Input/Output (RRIO) Op Amps http://www.intersil.com/ content/dam/Intersil/documents/isl2/isl28194-95.pdf

[12] TLV3691 0.9V to $6.5 \mathrm{~V}$, Nano-Power Comparator (Rev.C). http://www.ti.com/lit/ds/symlink/tlv3691.pdf

[13] Advandced Design System, Agilent Technologies. http://www.keysight.com/en/pc-1297113/ advanced-design-system-ads?cc=US\&lc=eng 